\title{
The Strict AKNS Hierarchy: Its Structure and Solutions
}

\author{
G. F. Helminck \\ Korteweg-de Vries Institute, University of Amsterdam, P.O. Box 94248, 1090 GE Amsterdam, Netherlands \\ Correspondence should be addressed to G. F. Helminck; g.f.helminck@uva.nl \\ Received 4 April 2016; Accepted 2 August 2016 \\ Academic Editor: Boris G. Konopelchenko \\ Copyright (C) 2016 G. F. Helminck. This is an open access article distributed under the Creative Commons Attribution License, \\ which permits unrestricted use, distribution, and reproduction in any medium, provided the original work is properly cited. \\ We discuss an integrable hierarchy of compatible Lax equations that is obtained by a wider deformation of a commutative algebra \\ in the loop space of $\mathrm{sl}_{2}$ than that in the AKNS case and whose Lax equations are based on a different decomposition of this loop \\ space. We show the compatibility of these Lax equations and that they are equivalent to a set of zero curvature relations. We present \\ a linearization of the system and conclude by giving a wide construction of solutions of this hierarchy.
}

\section{Introduction}

Integrable hierarchies often occur as the evolution equations of the generators of a deformation of a commutative subalgebra inside some Lie algebra $\mathfrak{g}$. The deformation and the evolution equations are determined by a splitting of $\mathfrak{g}$ in the direct sum of two Lie subalgebras, like in the AdlerKostant-Symes Theorem (see [1]). This gives then rise to a compatible set of Lax equations, a so-called hierarchy, and the simplest nontrivial equation often determines the name of the hierarchy. The hierarchy we discuss here corresponds to a somewhat different splitting of an algebra of $\mathrm{sl}_{2}$ loops that with respect to the usual decomposition yields the AKNS hierarchy. Following the terminology used in similar situations (see [2]), we use the name strict AKNS hierarchy for the system of Lax equations corresponding to this different decomposition.

The letters AKNS refer to the following fact: Ablowitz et al. showed (see [3]) that the initial value problem of the following system of equations for two complex functions $q$ and $r$ depending on the variables $x$ and $t$

$$
\begin{aligned}
& i \frac{\partial}{\partial t} q(x, t):=i q_{t}=-\frac{1}{2} q_{x x}+q^{2} r \\
& i \frac{\partial}{\partial t} r(x, t):=i r_{t}=\frac{1}{2} r_{x x}-q r^{2}
\end{aligned}
$$

could be solved with the Inverse Scattering Transform and that is why this system (1) is called the AKNS equations. It can be rewritten in a zero curvature form for $2 \times 2$-matrices as follows: consider the matrices

$$
\begin{aligned}
& P_{1}=\left(\begin{array}{ll}
0 & q \\
r & 0
\end{array}\right), \\
& P_{2}=\left(\begin{array}{cc}
-\frac{i}{2} q r & \frac{i}{2} q_{x} \\
-\frac{i}{2} r_{x} & \frac{i}{2} q r
\end{array}\right) .
\end{aligned}
$$

A direct computation shows that the following identities hold for them:

$$
\begin{aligned}
& \frac{\partial}{\partial x}\left(P_{2}\right)=\left(\begin{array}{cc}
\frac{i}{2}\left(q_{x} r+q r_{x}\right) & \frac{i}{2} q_{x x} \\
-\frac{i}{2} r_{x x} & -\frac{i}{2}\left(q_{x} r+q r_{x}\right)
\end{array}\right), \\
& {\left[P_{2}, P_{1}\right]=\left(\begin{array}{cc}
\frac{i}{2}\left(q_{x} r+q r_{x}\right) & i q^{2} r \\
-i q r^{2} & -\frac{i}{2}\left(q_{x} r+q r_{x}\right)
\end{array}\right) .}
\end{aligned}
$$

By combining them, one sees that the AKNS equations are equivalent to

$$
\frac{\partial}{\partial t}\left(P_{1}\right)-\frac{\partial}{\partial x}\left(P_{2}\right)-\left[P_{2}, P_{1}\right]=0 .
$$

In the next section, we will show that (4) is the simplest compatibility condition of a system of Lax equations that 
consequently carries the name AKNS hierarchy (see [4]). Besides that, we introduce here also another system of Lax equations for a more general deformation of the basic generator of the commutative algebra. It relates to a different decomposition of the relevant Lie algebra. Since its analogue for the KP hierarchy is called the strict KP hierarchy, we use in the present context the term strict AKNS hierarchy. We show first of all that this new system is compatible and equivalent with a set of zero curvature relations. Further, we describe suitable linearizations that can be used to construct solutions of both hierarchies. We conclude by constructing solutions of both hierarchies.

\section{The AKNS Hierarchy and Its Strict Version}

We present here an algebraic description of the AKNS hierarchy and its strict version that underlines the deformation character of these hierarchies as pointed at in Introduction. At (4), one has to deal with $2 \times 2$-matrices whose coefficients were polynomial expressions of a number of complex functions and their derivatives with respect to some parameters. The same is true for the other equations in both hierarchies. We formalize this as follows: our starting point is a commutative complex algebra $R$ that should be seen as the source from which the coefficients of the $2 \times 2$-matrices are taken. We will work in the Lie algebra $\mathrm{sl}_{2}(R)\left[z, z^{-1}\right)$ consisting of all elements

$$
X=\sum_{i=-\infty}^{N} X_{i} z^{i}, \quad \text { with all } X_{i} \in \mathrm{sl}_{2}(R)
$$

and the bracket

$$
\begin{aligned}
{[X, Y] } & =\left[\sum_{i=-\infty}^{N} X_{i} z^{i}, \sum_{j=-\infty}^{M} Y_{j} z^{j}\right] \\
& :=\sum_{i=-\infty}^{N} \sum_{j=-\infty}^{M}\left[X_{i}, Y_{j}\right] z^{i+j} .
\end{aligned}
$$

We will also make use of the slightly more general Lie algebra $\operatorname{gl}_{2}(R)\left[z, z^{-1}\right)$, where the coefficients in the $z$-series from (5) are taken from $\mathrm{gl}_{2}(R)$ instead of $\mathrm{sl}_{2}(R)$ and the bracket is given by the same formula. In the Lie algebra $\operatorname{gl}_{2}(R)\left[z, z^{-1}\right)$, we decompose elements in two ways. The first is as follows:

$$
X=\sum_{i=-\infty}^{N} X_{i} z^{i}=\sum_{i=0}^{N} X_{i} z^{i}+\sum_{i=-\infty}^{-1} X_{i} z^{i}=:(X)_{\geqslant 0}+(X)_{<0}
$$

and this induces the splitting

$$
\begin{aligned}
\operatorname{gl}_{2}(R)\left[z, z^{-1}\right)= & \mathrm{gl}_{2}(R)\left[z, z^{-1}\right)_{\geqslant 0} \\
& \oplus \mathrm{gl}_{2}(R)\left[z, z^{-1}\right)_{<0},
\end{aligned}
$$

where the two Lie subalgebras $\operatorname{gl}_{2}(R)\left[z, z^{-1}\right)_{\geqslant 0}$ and $\operatorname{gl}_{2}(R)[z$, $\left.z^{-1}\right)_{<0}$ are given by

$$
\begin{aligned}
\operatorname{gl}_{2} & (R)\left[z, z^{-1}\right)_{\geqslant 0} \\
& =\left\{X \in \operatorname{gl}_{2}(R)\left[z, z^{-1}\right) \mid X=(X)_{\geqslant 0}=\sum_{i=0}^{N} X_{i} z^{i}\right\},
\end{aligned}
$$

$$
\begin{aligned}
\operatorname{gl}_{2} & (R)\left[z, z^{-1}\right)_{<0} \\
& =\left\{X \in \operatorname{gl}_{2}(R)\left[z, z^{-1}\right) \mid X=(X)_{<0}=\sum_{i<0} X_{i} z^{i}\right\} .
\end{aligned}
$$

By restriction, it leads to a similar decomposition for $\operatorname{sl}_{2}(R)\left[z, z^{-1}\right)$, which is relevant for the AKNS hierarchy. The second way to decompose elements of $\operatorname{gl}_{2}(R)\left[z, z^{-1}\right)$ is

$$
\begin{aligned}
X & =\sum_{i=-\infty}^{N} X_{i} z^{i}=\sum_{i=1}^{N} X_{i} z^{i}+\sum_{i=-\infty}^{0} X_{i} z^{i} \\
& =(X)_{>0}+(X)_{\leqslant 0} .
\end{aligned}
$$

This yields the splitting

$$
\begin{aligned}
\operatorname{gl}_{2}(R)\left[z, z^{-1}\right)= & \operatorname{gl}_{2}(R)\left[z, z^{-1}\right)_{>0} \\
& \oplus \mathrm{gl}_{2}(R)\left[z, z^{-1}\right)_{\leqslant 0} .
\end{aligned}
$$

By restricting it to $\operatorname{sl}_{2}(R)\left[z, z^{-1}\right)$, we get a similar decomposition for this Lie algebra, which relates, as we will see further on, to the strict version of the AKNS hierarchy. The Lie subalgebras $\operatorname{gl}_{2}(R)\left[z, z^{-1}\right)_{>0}$ and $\operatorname{gl}_{2}(R)\left[z, z^{-1}\right)_{\leqslant 0}$ in (11) are defined in a similar way as the first two Lie subalgebras

$$
\begin{aligned}
\operatorname{gl}_{2} & (R)\left[z, z^{-1}\right)_{>0} \\
& =\left\{X \in \mathrm{gl}_{2}(R)\left[z, z^{-1}\right) \mid X=(X)_{>0}=\sum_{i=1}^{N} X_{i} z^{i}\right\}, \\
\mathrm{gl}_{2} & (R)\left[z, z^{-1}\right)_{\leqslant 0} \\
& =\left\{X \in \mathrm{gl}_{2}(R)\left[z, z^{-1}\right) \mid X=(X)_{\leqslant 0}=\sum_{i \leqslant 0} X_{i} z^{i}\right\} .
\end{aligned}
$$

Both hierarchies correspond to evolution equations for deformations of the generators of a commutative Lie algebra in the first component. Denote the matrix $\left(\begin{array}{cc}-i & 0 \\ 0 & i\end{array}\right)$ by $Q_{0}$. Inside $\mathrm{sl}_{2}(R)\left[z, z^{-1}\right)_{\geqslant 0}$, this commutative complex Lie subalgebra $C_{0}$ is chosen to be the Lie subalgebra with the basis $\left\{Q_{0} z^{m} \mid m \geqslant\right.$ $0\}$ and in $\mathrm{sl}_{2}(R)\left[z, z^{-1}\right)_{>0}$ our choice will be the Lie subalgebra $C_{1}$ with the basis $\left\{Q_{0} z^{m} \mid m \geqslant 1\right\}$. In the first case that we work with $C_{0}$, we assume that the algebra $R$ possesses a set $\left\{\partial_{m} \mid m \geqslant 0\right\}$ of commuting $\mathbb{C}$-linear derivations $\partial_{m}: R \rightarrow R$, where each $\partial_{m}$ should be seen as the derivation corresponding to the flow generated by $Q_{0} z^{m}$. The data $\left(R,\left\{\partial_{m} \mid m \geqslant 0\right\}\right)$ is also called a setting for the AKNS hierarchy. In the case that we work with the decomposition (11), we merely need a set of commuting $\mathbb{C}$-linear derivations $\partial_{m}: R \rightarrow R, m \geqslant 1$, with the same interpretation. Staying in the same line of terminology, we call the data $\left(R,\left\{\partial_{m} \mid m \geqslant 1\right\}\right)$ a setting for the strict AKNS hierarchy.

Example 1. Examples of settings for the respective hierarchies are the algebras of complex polynomials $\mathbb{C}\left[t_{m}\right]$ in the variables $\left\{t_{m} \mid m \geqslant 0\right\}$ and $\left\{t_{m} \mid m \geqslant 1\right\}$, respectively, or the 
formal power series $\mathbb{C}\left[\left[t_{m}\right]\right]$ in the same variables, with both algebras equipped with the derivations $\partial_{m}=\partial / \partial t_{m}, m \geqslant 0$, in the AKNS setup or $\partial_{m}=\partial / \partial t_{m}, m \geqslant 1$, in the strict AKNS case.

We let each derivation $\partial_{m}$ occurring in some setting act coefficient-wise on the matrices from $\mathrm{gl}_{2}(R)$ and that defines then a derivation of this algebra. The same holds for the extension to $\mathrm{gl}_{2}(R)\left[z, z^{-1}\right)$ defined by

$$
\partial_{m}(X):=\sum_{j=-\infty}^{N} \partial_{m}\left(X_{j}\right) z^{j}
$$

We have now sufficient ingredients to discuss the AKNS hierarchy and its strict version. In the AKNS case, our interest is in certain deformations of the Lie algebra $C_{0}$ obtained by conjugating with elements of the group corresponding to $\mathrm{gl}_{2}(R)\left[z, z^{-1}\right)_{<0}$. At the strict version, we are interested in certain deformations of the Lie algebra $C_{1}$ obtained by conjugating with elements from a group linked to $\mathrm{gl}_{2}(R)\left[z, z^{-1}\right)_{\leqslant 0}$.

Note that for each $X \in \mathrm{gl}_{2}(R)\left[z, z^{-1}\right)_{<0}$ the exponential map yields a well-defined element of the form

$$
\exp (X)=\sum_{k=0}^{\infty} \frac{1}{k !} X^{k}=\mathrm{Id}+Y, \quad Y \in \mathrm{gl}_{2}(R)\left[z, z^{-1}\right)_{<0}
$$

and with the formula for the logarithm one retrieves $X$ back from $Y$. One verifies directly that the elements of the form (14) form a group with respect to multiplication and this we see as the group $G_{<0}$ corresponding to $\mathrm{gl}_{2}(R)\left[z, z^{-1}\right)_{<0}$.

In the case of the Lie subalgebra $\operatorname{gl}_{2}(R)\left[z, z^{-1}\right)_{\leqslant 0}$, one cannot move back and forth between the Lie algebra and its group. Nevertheless, one can assign a proper group to this Lie algebra. A priori, the exponential $\exp (Y)$ of an element $Y \in \operatorname{gl}_{2}(R)\left[z, z^{-1}\right)_{\leqslant 0}$ does not have to define an element in $\operatorname{gl}_{2}(R)\left[z, z^{-1}\right)_{\leqslant 0}$. That requires convergence conditions. However, if it does, then it belongs to

$$
\begin{aligned}
& G_{\leqslant 0}=\left\{K=\sum_{j=0}^{\infty} K_{j} z^{-j} \mid \text { all } K_{j} \in \mathrm{gl}_{2}(R), K_{0}\right. \\
& \left.\quad \in \operatorname{gl}_{2}(R)^{*}\right\},
\end{aligned}
$$

where $\mathrm{gl}_{2}(R)^{*}$ denotes the elements in $\mathrm{gl}_{2}(R)$ that have a multiplicative inverse in $\mathrm{gl}_{2}(R)$. It is a direct verification that $G_{\leqslant 0}$ is a group and we see it as a proper group corresponding to the Lie algebra $\operatorname{gl}_{2}(R)\left[z, z^{-1}\right)_{\leqslant 0}$. In fact, $G_{\leqslant 0}$ is isomorphic to the semidirect product of $G_{<0}$ and $\mathrm{gl}_{2}(R)^{*}$.

Now there holds the following.

Lemma 2. The group $G_{\leqslant 0}$ acts by conjugation on $\operatorname{sl}_{2}(R)[z$, $\left.z^{-1}\right)$.
Proof. Take first any $g \in G_{<0}$. Then, there is an $X \in \mathrm{gl}_{2}(R)[z$, $\left.z^{-1}\right)_{<0}$ such that $g=\exp (X)$. Now there holds for every $Y \in$ $\mathrm{sl}_{2}(R)\left[z, z^{-1}\right)$

$$
\begin{aligned}
g Y g^{-1} & =\exp (X) Y \exp (-X)=e^{\operatorname{ad}(X)}(Y) \\
& =Y+\sum_{k=1}^{\infty} \frac{1}{k !} \operatorname{ad}(X)^{k}(Y)
\end{aligned}
$$

and this shows that the coefficients for the different powers of $z$ in this expression are commutators of elements of $\mathrm{gl}_{2}(R)$ and $\mathrm{sl}_{2}(R)$ and that proves the claim for elements from $G_{<0}$. Since conjugation with an element from $\mathrm{gl}_{2}(R)^{*}$ maps $\mathrm{sl}_{2}(R)$ to itself, the same holds for $\mathrm{sl}_{2}(R)\left[z, z^{-1}\right)$. This proves the full claim.

Next we have a look at the different deformations. The deformations of $C_{0}$ by elements of $G_{<0}$ are determined by that of $Q_{0}$. Therefore, we focus on that element and we consider for some $g=\exp (X)=\exp \left(\sum_{j=1}^{\infty} X_{j} z^{-j}\right) \in G_{<0}$ the deformation

$$
\begin{aligned}
Q= & g Q_{0} g^{-1}:=\sum_{i=0}^{\infty} Q_{i} z^{-i} \\
= & \left(\begin{array}{cc}
-i & 0 \\
0 & i
\end{array}\right)+\left[X_{1}, Q_{0}\right] z^{-1} \\
& +\left\{\left[X_{2}, Q_{0}\right]+\frac{1}{2}\left[X_{1},\left[X_{1}, Q_{0}\right]\right]\right\} z^{-2}+\cdots
\end{aligned}
$$

of $Q_{0}$. From this formula, we see directly that if each $X_{i}=$ $\left(\begin{array}{cc}-\alpha_{i} & \beta_{i} \\ \gamma_{i} & \alpha_{i}\end{array}\right), i=1,2$, then

$$
\begin{aligned}
Q_{1} & =\left(\begin{array}{cc}
0 & 2 i \beta_{1} \\
-2 i \gamma_{i} & 0
\end{array}\right)=\left(\begin{array}{ll}
0 & q \\
r & 0
\end{array}\right), \\
Q_{2} & =\left(\begin{array}{cc}
q_{11} & q_{12} \\
q_{21} & q_{22}
\end{array}\right) \\
& =\left(\begin{array}{cc}
-2 i \beta_{1} \gamma_{1} & 2 i\left(\beta_{2}-\alpha_{1} \beta_{1}\right) \\
-2 i\left(\gamma_{2}+\alpha_{1} \gamma_{1}\right) & 2 i \beta_{1} \gamma_{1}
\end{array}\right) .
\end{aligned}
$$

In particular, we get in this way that $q_{11}=-i(q r / 2)$ and $q_{22}=i(q r / 2)$. Since $Q$ is the deformation of $Q_{0}$ and $\operatorname{Id} z$ is central, the deformation of each $Q_{0} z^{m}, m \in \mathbb{Z}$, is given by $\mathrm{Q} z^{m}$. The deformation of the Lie algebra $C_{1}$ by elements from $G_{\leqslant 0}$ is basically determined by that of the element $Q_{0} z$. So we focus on the deformation of this element. Using the same notations as at the deformation of $Q_{0}$ by $G_{<0}$, we get that the deformation of $Q_{0} z$ by a $K g \in G_{\leqslant 0}$, with $K \in \mathrm{gl}_{2}(R)^{*}$ and $g \in G_{<0}$, looks like

$$
\begin{aligned}
Z & =K g Q_{0} z g^{-1} K^{-1}:=\sum_{i=0}^{\infty} K Q_{i} K^{-1} z^{1-i}=\sum_{i=0}^{\infty} Z_{i} z^{1-i} \\
& =Z_{0} z+\left[K X_{1} K^{-1}, Z_{0}\right]+\cdots .
\end{aligned}
$$

Consequently, the corresponding deformation of each $Q_{0} z^{m}$, $m \geqslant 1$, is $Z z^{m-1}$. 
Now we are looking for deformations $Q$ of the form (17) such that the evolution with respect to the $\left\{\partial_{m}\right\}$ satisfies the following: for all $m \geqslant 0$,

$$
\partial_{m}(Q)=\left[\left(Q z^{m}\right)_{\geqslant 0}, Q\right]=-\left[\left(Q z^{m}\right)_{<0}, Q\right],
$$

where the second identity follows from the fact that all $\left\{Q z^{m}\right\}$ commute. Equations (20) are called the Lax equations of the AKNS hierarchy and the deformation $Q$ satisfying these equations is called a solution of the hierarchy. Note that $Q=Q_{0}$ is a solution of the AKNS hierarchy and it is called the trivial one. AKNS equation (4) occurs among the compatibility equations of this system, as we will see further on. Note that $(20)$ for $m=0$ is simply $\partial_{0}(Q)=\left[Q_{0}, Q\right]$. Therefore, if $\partial_{0}=\partial / \partial t_{0}$ and both $e^{-i t_{0}}$ and $e^{i t_{0}}$ belong to the algebra $R$ of matrix coefficients, then we can introduce the loop $\widehat{Q} \in \operatorname{sl}_{2}(R)\left[z, z^{-1}\right)$ given by

$$
\begin{aligned}
\widehat{Q} & :=\exp \left(-t_{0} Q_{0}\right) Q \exp \left(t_{0} Q_{0}\right) \\
& =\sum_{k \geqslant 0} \exp \left(-t_{0} Q_{0}\right) Q_{k} \exp \left(t_{0} Q_{0}\right) z^{-k}
\end{aligned}
$$

which is easily seen to satisfy $\partial_{0}(\widehat{Q})=0$. This handles then the dependence of $Q$ of $t_{0}$.

Among the deformations $Z$ of the form (19), we look for $Z$ such that their evolution with respect to $\left\{\partial_{m}\right\}$ satisfies the following: for all $m \geqslant 1$,

$$
\partial_{m}(Z)=\left[\left(Z z^{m-1}\right)_{>0}, Z\right]=-\left[\left(Z z^{m-1}\right)_{\leqslant 0}, Z\right],
$$

where the second identity follows from the fact that all $\left\{Z z^{m-1}\right\}$ commute. Since (22) correspond to the strict cutoff (10), they are called the Lax equations of the strict AKNS hierarchy and the deformation $Z$ is called a solution of this hierarchy. Again there is at least one solution $Z=Q_{0} z$. It is called the trivial solution of the hierarchy.

For both systems (20) and (22), one can speak of compatibility. Namely, there holds the following result.

Proposition 3. Both sets of Lax equations (20) and (22) are the so-called compatible systems; that is, the projections $\left\{B_{m}:=\right.$ $\left.\left(Q z^{m}\right)_{\geqslant 0} \mid m \geqslant 0\right\}$ of a solution $Q$ to (20) satisfy the zero curvature relations

$$
\partial_{m_{1}}\left(B_{m_{2}}\right)-\partial_{m_{2}}\left(B_{m_{1}}\right)-\left[B_{m_{1}}, B_{m_{2}}\right]=0
$$

and the projections $\left\{C_{m}:=\left(Z z^{m-1}\right)_{>0} \mid m \geqslant 1\right\}$ corresponding to a solution $Z$ to (22) satisfy the zero curvature relations

$$
\partial_{m_{1}}\left(C_{m_{2}}\right)-\partial_{m_{2}}\left(C_{m_{1}}\right)-\left[C_{m_{1}}, C_{m_{2}}\right]=0
$$

Proof. The idea is to show that the left-hand side of (23) and (24), respectively, belongs to

$$
\operatorname{sl}_{2}(R)\left[z, z^{-1}\right)_{\geqslant 0} \cap \operatorname{sl}_{2}(R)\left[z, z^{-1}\right)_{<0}
$$

respectively $\mathrm{sl}_{2}(R)\left[z, z^{-1}\right)_{>0} \cap \mathrm{sl}_{2}(R)\left[z, z^{-1}\right)_{\leqslant 0}$ and thus has to be zero. We give the proof for $\left\{C_{m}\right\}$; that for $\left\{B_{m}\right\}$ is similar and is left to the reader. The inclusion in the first factor is clear as both $C_{m}$ and $\partial_{n}\left(C_{m}\right)$ belong to the Lie subalgebra $\mathrm{sl}_{2}(R)\left[z, z^{-1}\right)_{>0}$. To show the other one, we use Lax equations (22). Note that the same Lax equations hold for all the $\left\{z^{N} Z \mid N \geqslant 0\right\}$ :

$$
\partial_{m}\left(z^{N} Z\right)=\left[\left(Z z^{m-1}\right)_{>0}, z^{N} Z\right] .
$$

By substituting $C_{m_{i}}=z^{m_{i}-1} Z-\left(z^{m_{i}-1} Z\right)_{<0}$, we get the identities for

$$
\begin{aligned}
\partial_{m_{1}}( & \left.C_{m_{2}}\right)-\partial_{m_{2}}\left(C_{m_{1}}\right) \\
= & \partial_{m_{1}}\left(z^{m_{2}-1} Z\right)-\partial_{m_{1}}\left(\left(z^{m_{2}-1} Z\right)_{\leqslant 0}\right) \\
& -\partial_{m_{2}}\left(z^{m_{1}-1} Z\right)+\partial_{m_{2}}\left(\left(z^{m_{1}-1} Z\right)_{\leqslant 0}\right) \\
= & {\left[C_{m_{1}}, z^{m_{2}-1} Z\right]-\left[C_{m_{2}}, z^{m_{1}-1} Z\right] } \\
& -\partial_{m_{1}}\left(\left(z^{m_{2}-1} Z\right)_{\leqslant 0}\right)+\partial_{m_{2}}\left(\left(z^{m_{1}-1} Z\right)_{\leqslant 0}\right)
\end{aligned}
$$

and

$$
\begin{aligned}
{\left[C_{m_{1}}, C_{m_{2}}\right] } & \\
= & {\left[z^{m_{1}-1} Z-\left(z^{m_{1}-1} Z\right)_{\leqslant 0}, z^{m_{2}-1} Z-\left(z^{m_{2}-1} Z\right)_{\leqslant 0}\right] } \\
= & -\left[\left(z^{m_{1}-1} Z\right)_{\leqslant 0}, z^{m_{2}-1} Z\right] \\
& +\left[\left(z^{m_{2}-1} Z\right)_{\leqslant 0}, z^{m_{1}-1} Z\right] \\
& +\left[\left(z^{m_{1}-1} Z\right)_{\leqslant 0},\left(z^{m_{2}-1} Z\right)_{\leqslant 0}\right] .
\end{aligned}
$$

Taking into account the second identity in (22), we see that the left-hand side of (24) is equal to

$$
\begin{gathered}
-\partial_{m_{1}}\left(\left(z^{m_{2}-1} Z\right)_{\leqslant 0}\right)+\partial_{m_{2}}\left(\left(z^{m_{1}-1} Z\right)_{\leqslant 0}\right) \\
-\left[\left(z^{m_{1}-1} Z\right)_{\leqslant 0},\left(z^{m_{2}-1} Z\right)_{\leqslant 0}\right] .
\end{gathered}
$$

This element belongs to the Lie subalgebra $\mathrm{sl}_{2}(R)\left[z, z^{-1}\right)_{\leqslant 0}$ and that proves the claim.

Reversely, we have the following.

Proposition 4. Suppose we have a deformation $Q$ of the type (17) or a deformation $Z$ of the form (19). Then, there holds the following:

(1) Assume that the projections $\left\{B_{m}:=\left(Q z^{m}\right)_{\geqslant 0} \mid m \geqslant 0\right\}$ satisfy the zero curvature relations (23). Then, $Q$ is a solution of the AKNS hierarchy.

(2) Similarly, if the projections $\left\{C_{m}:=\left(Z z^{m-1}\right)_{\geqslant 0} \mid m \geqslant\right.$ $1\}$ satisfy the zero curvature relations (24), then $Z$ is a solution of the strict AKNS hierarchy. 
Proof. Again we prove the statement for $Z$; that for $Q$ is shown in a similar way. So, assume that there is one Lax equation (22) that does not hold. Then, there is a $m \geqslant 1$ such that

$$
\partial_{m}(Z)-\left[C_{m}, Z\right]=\sum_{j \leqslant k(m)} X_{j} z^{j}, \quad \text { with } X_{k(m)} \neq 0 .
$$

Since both $\partial_{m}(Z)$ and $\left[C_{m}, Z\right]$ are of order smaller than or equal to one in $z$, we know that $k(m) \leqslant 1$. Further, we can say that for all $N \geqslant 0$

$$
\partial_{m}\left(z^{N} Z\right)-\left[C_{m}, z^{N} Z\right]=\sum_{j \leqslant k(m)} X_{j} z^{j+N},
$$

$$
\text { with } X_{k(m)} \neq 0
$$

and we see by letting $N$ go to infinity that the right-hand side can obtain any sufficiently large order in $z$. By the zero curvature relation for $N$ and $m$, we get for the left-hand side

$$
\begin{aligned}
\partial_{m}\left(z^{N} Z\right)-\left[C_{m}, z^{N} Z\right] \\
=\partial_{m}\left(C_{N}\right)-\left[C_{m}, C_{N}\right]+\partial_{m}\left(\left(z^{N} Z\right)_{\leqslant 0}\right) \\
\quad-\left[C_{m},\left(z^{N} Z\right)_{\leqslant 0}\right] \\
=\partial_{N}\left(C_{m}\right)+\partial_{m}\left(\left(z^{N} Z\right)_{\leqslant 0}\right)-\left[C_{m},\left(z^{N} Z\right)_{\leqslant 0}\right]
\end{aligned}
$$

and this last expression is of order smaller or equal to $m$ in $z$. This contradicts the unlimited growth in orders of $z$ of the right-hand side. Hence, all Lax equations (22) have to hold for $Z$.

Because of the equivalence between Lax equations (20) for $Q$ and the zero curvature relations (23) for $\left\{B_{m}\right\}$, we call this last set of equations also the zero curvature form of the AKNS hierarchy. Similarly, the zero curvature relations (24) for $\left\{C_{m}\right\}$ are called the zero curvature form of the strict AKNS hierarchy.

Besides the zero curvature relations for the cut-off's $\left\{B_{m}\right\}$ and $\left\{C_{m}\right\}$, respectively, corresponding to, respectively, a solution $Q$ of the AKNS hierarchy and a solution $Z$ of the strict AKNS hierarchy, also other parts satisfy such relations. Define

$$
\begin{aligned}
& A_{m}:=B_{m}-Q z^{m}, \quad m \geqslant 0, \\
& D_{m}:=C_{m}-Z z^{m-1}, \quad m \geqslant 1 .
\end{aligned}
$$

Then, we have the following result.

Corollary 5. The following relations hold:

(i) The parts $\left\{A_{m} \mid m \geqslant 0\right\}$ of a solution $Q$ of the AKNS hierarchy satisfy

$$
\partial_{m_{1}}\left(A_{m_{2}}\right)-\partial_{m_{2}}\left(A_{m_{1}}\right)-\left[A_{m_{1}}, A_{m_{2}}\right]=0 .
$$

(ii) The parts $\left\{D_{m} \mid m \geqslant 0\right\}$ of a solution $Z$ of the strict AKNS hierarchy satisfy

$$
\partial_{m_{1}}\left(D_{m_{2}}\right)-\partial_{m_{2}}\left(D_{m_{1}}\right)-\left[D_{m_{1}}, D_{m_{2}}\right]=0
$$

Proof. Again we show the result only in the strict case. Note that $\left\{Z z^{m-1}\right\}$ satisfy Lax equations similar to $Z$ :

$$
\partial_{i}\left(Z z^{m-1}\right)=\left[D_{i}, Z z^{m-1}\right], \quad i \geqslant 1 .
$$

Now we substitute in the zero curvature relations for $\left\{C_{m}\right\}$ everywhere the relation $C_{m}=D_{m}+Z z^{m-1}$ and use the Lax equations above and the fact that all $\left\{Z z^{m-1}\right\}$ commute. This gives the desired result.

To clarify the link with the AKNS equation, consider relation (23) for $m_{1}=2$ and $m_{2}=1$ :

$$
\begin{aligned}
\partial_{2}\left(Q_{0} z+Q_{1}\right)= & \partial_{1}\left(Q_{0} z^{2}+Q_{1} z+Q_{2}\right) \\
& +\left[Q_{2}, Q_{0} z+Q_{1}\right] .
\end{aligned}
$$

Then, this identity reduces in $\operatorname{sl}_{2}(R)\left[z, z^{-1}\right)_{\geqslant 0}$, since $Q_{0}$ is constant, to the following two equalities:

$$
\begin{aligned}
& \partial_{1}\left(Q_{1}\right)=\left[Q_{0}, Q_{2}\right] \\
& \partial_{2}\left(Q_{1}\right)=\partial_{1}\left(Q_{2}\right)+\left[Q_{2}, Q_{1}\right] .
\end{aligned}
$$

The first gives an expression of the off-diagonal terms of $Q_{2}$ in the coefficients $q$ and $r$ of $Q_{1}$; that is,

$$
\begin{aligned}
& q_{12}=\frac{i}{2} \partial_{1}(q), \\
& q_{21}=-\frac{i}{2} \partial_{1}(r),
\end{aligned}
$$

and the second equation becomes AKNS equations (4), if one has $\partial_{1}=\partial / \partial x$ and $\partial_{2}=\partial / \partial t$.

\section{The Linearization of Both Hierarchies}

The zero curvature form of both hierarchies points at the possible existence of a linear system of which the zero curvature equations form the compatibility conditions. We present here such a system for each hierarchy. For the AKNS hierarchy, this system, the linearization of the AKNS hierarchy, is as follows: take a potential solution $Q$ of the form (17) and consider the system

$$
\begin{aligned}
Q \psi & =\psi Q_{0}, \\
\partial_{m}(\psi) & =B_{m} \psi, \quad \forall m \geqslant 0, B_{m}=\left(Q z^{m}\right)_{\geqslant 0} .
\end{aligned}
$$

Likewise, for a potential solution $Z$ of the strict AKNS hierarchy of the form (19), the linearization of the strict AKNS hierarchy is given by

$$
\begin{aligned}
Z \varphi & =\varphi Q_{0} z, \\
\partial_{m}(\varphi) & =C_{m} \varphi, \quad \forall m \geqslant 1, C_{m}=\left(Z z^{m-1}\right)_{>0} .
\end{aligned}
$$

Before specifying $\psi$ and $\varphi$, we show the manipulations needed to derive the Lax equations of the hierarchy from their linearization. We do this for the strict AKNS hierarchy; for the 
AKNS hierarchy one proceeds similarly. Apply $\partial_{m}$ to the first equation in (41) and use both equalities in (41) in the sequel:

$$
\begin{aligned}
\partial_{m}\left(Z \varphi-\varphi Q_{0} z\right) & =\partial_{m}(Z) \varphi+Z \partial_{m}(\varphi)-\partial_{m}(\varphi) Q_{0} z \\
& =0=\partial_{m}(Z) \varphi+Z C_{m} \varphi-C_{m} \varphi Q_{0} z \\
& =\left\{\partial_{m}(Z)-\left[C_{m}, Z\right]\right\} \varphi=0 .
\end{aligned}
$$

Hence, if we can scratch $\varphi$ from (42), then we obtain the Lax equations of the strict AKNS hierarchy. To get the proper setup where all these manipulations make sense, we first have a look at the linearization for the trivial solutions $Q=Q_{0}$ and $Z=Q_{0} z$. In the AKNS case, we have then

$$
\begin{aligned}
Q_{0} \psi_{0} & =\psi_{0} Q_{0}, \\
\partial_{m}\left(\psi_{0}\right) & =Q_{0} z^{m} \psi_{0}, \quad \forall m \geqslant 0
\end{aligned}
$$

and for its strict version

$$
\begin{aligned}
Q_{0} z \varphi_{0} & =\varphi_{0} Q_{0} z, \\
\partial_{m}\left(\varphi_{0}\right) & =Q_{0} z^{m} \varphi_{0}, \quad \forall m \geqslant 1 .
\end{aligned}
$$

Assuming that each derivation $\partial_{m}$ equals $\partial / \partial t_{m}$, one arrives for (43) at the solution

$$
\psi_{0}=\psi_{0}(t, z)=\exp \left(\sum_{m=0}^{\infty} t_{m} Q_{0} z^{m}\right),
$$

where $t$ is the short hand notation for $\left\{t_{m} \mid m \geqslant 0\right\}$, and for (44) it leads to

$$
\begin{aligned}
& \varphi_{0}=\varphi_{0}(t, z)=\exp \left(\sum_{m=1}^{\infty} t_{m} Q_{0} z^{m}\right), \\
& \text { with } t=\left\{t_{m} \mid m \geqslant 1\right\} .
\end{aligned}
$$

In general, one needs in the linearizations $(\psi)$ and $(\varphi)$, respectively, a left action with elements like $Q, B_{m}$ and $Z$, $C_{m}$ from $\mathrm{gl}_{2}(R)\left[z, z^{-1}\right)$, respectively, an action of all the $\partial_{m}$, and a right action of $Q_{0}$ and $Q_{0} z$, respectively. This can all be realized in suitable $\mathrm{gl}_{2}(R)\left[z, z^{-1}\right)$ modules that are perturbations of the trivial solutions $\psi_{0}$ and $\varphi_{0}$. Consider in a AKNS setting

$$
\begin{aligned}
\mathscr{M}_{0} & =\left\{\{g(z)\} \psi_{0}=\left\{\sum_{i=-\infty}^{N} g_{i} z^{i}\right\} \psi_{0} \mid g(z)\right. \\
& \left.=\sum_{i=-\infty}^{N} g_{i} z^{i} \in \mathrm{gl}_{2}(R)\left[z, z^{-1}\right)\right\}
\end{aligned}
$$

and in a strict AKNS setting

$$
\begin{aligned}
\mathscr{M}_{1} & =\left\{\{g(z)\} \varphi_{0}=\left\{\sum_{i=-\infty}^{N} g_{i} z^{i}\right\} \varphi_{0} \mid g(z)\right. \\
& \left.=\sum_{i=-\infty}^{N} g_{i} z^{i} \in \mathrm{gl}_{2}(R)\left[z, z^{-1}\right)\right\},
\end{aligned}
$$

where the products $\{g(z)\} \psi_{0}$ and $\{g(z)\} \varphi_{0}$ should be seen as formal and both factors should be kept separate. On both $\mathscr{M}_{0}$ and $\mathscr{M}_{1}$, one can define the required actions: for each $h(z) \in$ $\mathrm{gl}_{2}(R)\left[z, z^{-1}\right)$, define

$$
h(z) \cdot\{g(z)\} \psi_{0}:=\{h(z) g(z)\} \psi_{0}
$$

respectively $h(z) \cdot\{g(z)\} \varphi_{0}:=\{h(z) g(z)\} \varphi_{0}$.

We define the right-hand action of $Q_{0}$ and $Q_{0} z$, respectively, by

$$
\begin{array}{r}
\{g(z)\} \psi_{0} Q_{0}:=\left\{g(z) Q_{0}\right\} \psi_{0} \\
\text { respectively }\{g(z)\} \varphi_{0} Q_{0} z:=\left\{g(z) Q_{0} z\right\} \varphi_{0}
\end{array}
$$

and the action of each $\partial_{m}$ by

$$
\begin{aligned}
\partial_{m} & \left(\{g(z)\} \psi_{0}\right) \\
= & \left\{\sum_{i=-\infty}^{N} \partial_{m}\left(g_{i}\right) z^{i}+\left\{\sum_{i=-\infty}^{N} g_{i} Q_{0} z^{i+m}\right\}\right\} \psi_{0}, \\
\partial_{m} & \left(\{g(z)\} \varphi_{0}\right) \\
= & \left\{\sum_{i=-\infty}^{N} \partial_{m}\left(g_{i}\right) z^{i}+\left\{\sum_{i=-\infty}^{N} g_{i} Q_{0} z^{i+m}\right\}\right\} \varphi_{0} .
\end{aligned}
$$

Analogous to the terminology in the scalar case (see [5]), we call the elements of $\mathscr{M}_{0}$ and $\mathscr{M}_{1}$ oscillating matrices. Note that both $\mathscr{M}_{0}$ and $\mathscr{M}_{1}$ are free $\mathrm{gl}_{2}(R)\left[z, z^{-1}\right)$ modules with generators $\psi_{0}$ and $\varphi_{0}$, respectively, because for each $h(z) \epsilon$ $\mathrm{gl}_{2}(R)\left[z, z^{-1}\right)$ we have

$$
h(z) \cdot \psi_{0}=h(z) \cdot\{1\} \psi_{0}=\{h(z)\} \psi_{0},
$$

respectively $h(z) \cdot \varphi_{0}=h(z) \cdot\{1\} \psi_{0}=\{h(z)\} \varphi_{0}$.

Hence, in order to be able to perform legally the scratching of $\psi=\{h(z)\} \psi_{0}$ or $\varphi=\{h(z)\} \varphi_{0}$, it is enough to find oscillating matrices such that $h(z)$ is invertible in $\operatorname{gl}_{2}(R)\left[z, z^{-1}\right)$. We will now introduce a collection of such elements that will occur at the construction of solutions of both hierarchies. by

For $m=\left(m_{1}, m_{2}\right) \in \mathbb{Z}^{2}$, let $\delta(m) \in \mathrm{gl}_{2}(R)\left[z, z^{-1}\right)$ be given

$$
\delta(m)=\left(\begin{array}{cc}
z^{m_{1}} & 0 \\
0 & z^{m_{2}}
\end{array}\right)
$$

Then, $\delta(m)$ has $\delta(-m)$ as its inverse in $\mathrm{gl}_{2}(R)\left[z, z^{-1}\right)$ and the collection $\Delta=\left\{\delta(m) \mid m \in \mathbb{Z}^{2}\right\}$ forms a group. An element $\psi \in \mathscr{M}_{0}$ is called an oscillating matrix of type $\delta(m)$ if it has the form

$$
\psi=\{h(z) \delta(m)\} \psi_{0}, \quad \text { with } h(z) \in G_{<0} .
$$

These oscillating matrices are examples of elements of $\mathscr{M}_{0}$ for which the scratching procedure is valid. Let $Q$ be a potential solution of the AKNS hierarchy of the form (17). If there is an oscillating function $\psi$ of type $\delta(m)$ such that for $Q$ and $\psi$ 
(40) hold, then we call $\psi$ a wave matrix of the AKNS hierarchy of type $\delta(m)$. In particular, $Q$ is then a solution of the AKNS hierarchy and the first equation in (40) implies

$$
\begin{aligned}
Q h(z) \delta(m) & =h(z) Q_{0} \delta(m) \Longrightarrow \\
Q & =h(z) Q_{0} h(z)^{-1} ;
\end{aligned}
$$

in other words, the solution $Q$ is totally determined by the wave matrix. Similarly, we call an element $\varphi \in \mathscr{M}_{1}$ an oscillating matrix of type $\delta(m)$ if it has the form

$$
\varphi=\{h(z) \delta(m)\} \varphi_{0}, \quad \text { with } h(z) \in G_{\leqslant 0} .
$$

This type of oscillating matrices are examples of elements of $\mathscr{M}_{1}$ for which the scratching procedure is valid. Let $Z$ be a potential solution of the strict AKNS hierarchy of the form (19). If there is an oscillating function $\varphi$ of type $\delta(m)$ in $\mathscr{M}_{1}$ such that for $Z$ and $\varphi$ (41) hold, then we call $\varphi$ a wave matrix of the strict AKNS hierarchy of type $\delta(m)$. In particular, $Z$ is then a solution of the strict AKNS hierarchy and the first equation in (41) implies

$$
\begin{aligned}
Z h(z) \delta(m) & =h(z) Q_{0} z \delta(m) \Longrightarrow \\
Z & =h(z) Q_{0} z h(z)^{-1},
\end{aligned}
$$

so that also here the wave matrix fully determines the solution.

For both hierarchies, there is a milder condition that is to be satisfied by oscillating matrices of a certain type, in order that they become a wave matrix of that hierarchy.

Proposition 6. Let $\psi=\{h(z) \delta(m)\} \psi_{0}$ be an oscillating matrix of type $\delta(m)$ in $\mathscr{M}_{0}$ and $Q=h(z) Q_{0} h(z)^{-1}$ the corresponding potential solution of the AKNS hierarchy. Similarly, let $\varphi=$ $\{h(z) \delta(m)\} \varphi_{0}$ be such a matrix in $\mathscr{M}_{1}$ with potential solution $Z=h(z) Q_{0} z h(z)^{-1}$.

(a) If there exists for each $m \geqslant 0$ an element $M_{m} \in$ $\mathrm{gl}_{2}(R)\left[z, z^{-1}\right)_{\geqslant 0}$ such that

$$
\partial_{m}(\psi)=M_{m} \psi
$$

then each $M_{m}=\left(Q z^{m}\right)_{\geqslant 0}$ and $\psi$ is a wave matrix of type $\delta(m)$ for the AKNS hierarchy.

(b) If there exists for each $m \geqslant 1$ an element $N_{m} \in$ $\mathrm{gl}_{2}(R)\left[z, z^{-1}\right)_{>0}$ such that

$$
\partial_{m}(\varphi)=N_{m} \varphi
$$

then each $N_{m}=\left(Z z^{m-1}\right)_{>0}$ and $\varphi$ is a wave matrix of type $\delta(m)$ for the strict AKNS hierarchy.

Proof. We give the proof again for the strict AKNS case; the other one is similar. By using the fact that $\mathscr{M}_{1}$ is a free $\operatorname{gl}_{2}(R)\left[z, z^{-1}\right)$ module with generator $\varphi_{0}$ we can translate the relations $\partial_{m}(\varphi)=N_{m} \varphi$ into equations in $\operatorname{gl}_{2}(R)\left[z, z^{-1}\right)$. This yields

$$
\begin{aligned}
\partial_{m}(h(z))+h(z) Q_{0} z^{m} & =N_{m} h(z) \Longrightarrow \\
\partial_{m}(h(z)) h(z)^{-1}+Z z^{m-1} & =N_{m} .
\end{aligned}
$$

Projecting the right-hand side on $\operatorname{gl}_{2}(R)\left[z, z^{-1}\right)_{>0}$ gives us the identity

$$
\left(Z z^{m-1}\right)_{>0}=N_{m}
$$

we are looking for.

In the next section, we produce a context where we can construct wave matrices for both hierarchies in which the product is real.

\section{The Construction of the Solutions}

In this section, we will show how to construct a wide class of solutions of both hierarchies. This is done in the style of [6]. We first describe the group of loops we will work with. For each $0<r<1$, let $A_{r}$ be the annulus

$$
\left\{z|z \in \mathbb{C}, r \leqslant| z \mid \leqslant \frac{1}{r}\right\} .
$$

Following [7], we use the notation $L_{\text {an }} \mathrm{GL}_{2}(\mathbb{C})$ for the collection of holomorphic maps from some annulus $A_{r}$ into $\mathrm{GL}_{2}(\mathbb{C})$. It is a group with respect to pointwise multiplication and contains in a natural way $\mathrm{GL}_{2}(\mathbb{C})$ as a subgroup as the collection of constant maps into $\mathrm{GL}_{2}(\mathbb{C})$. Other examples of elements in $L_{\text {an }} \mathrm{GL}_{2}(\mathbb{C})$ are the elements of $\Delta$. However, $L_{\text {an }} \mathrm{GL}_{2}(\mathbb{C})$ is more than just a group; it is an infinite dimensional Lie group. Its manifold structure can be read off from its Lie algebra $L_{\text {angl }}(\mathbb{C})$ consisting of all holomorphic maps $\gamma: U \rightarrow \mathrm{gl}_{2}(\mathbb{C})$, where $U$ is an open neighborhood of some annulus $A_{r}, 0<r<1$. Since $\mathrm{gl}_{2}(\mathbb{C})$ is a Lie algebra, the space $L_{\text {an }} \mathrm{gl}_{2}(\mathbb{C})$ becomes a Lie algebra with respect to the pointwise commutator. Topologically, the space $L_{\text {an }} \mathrm{gl}_{2}(\mathbb{C})$ is the direct limit of all the spaces $L_{\text {an, } r} \mathrm{gl}_{2}(\mathbb{C})$, where this last space consists of all $\gamma$ corresponding to the fixed annulus $A_{r}$. One gives each $L_{\mathrm{an}, r} \mathrm{gl}_{2}(\mathbb{C})$ the topology of uniform convergence and with that topology it becomes a Banach space. In this way, $L_{\text {an }} \mathrm{gl}_{2}(\mathbb{C})$ becomes a Fréchet space. The pointwise exponential map defines a local diffeomorphism around zero in $L_{\text {angl }}(\mathbb{C})$ (see, e.g., [8]).

Now each $\gamma \in L_{\text {angl }}(\mathbb{C})$ possesses an expansion in a Fourier series

$$
\gamma=\sum_{k=-\infty}^{\infty} \gamma_{k} z^{k}, \quad \text { with each } \gamma_{k} \in \mathrm{gl}_{2}(\mathbb{C})
$$

that converges absolutely on the annulus $A_{r}$ :

$$
\sum_{k=-\infty}^{\infty}\left\|\gamma_{k}\right\| r^{-|k|}<\infty
$$

This Fourier expansion is used to make two different decompositions of the Lie algebra $L_{\text {angl }}(\mathbb{C})$. The first is the analogue for the present Lie algebra $L_{\text {an }} \mathrm{gl}_{2}(\mathbb{C})$ of decomposition (8) of $\operatorname{gl}_{2}(R)\left[z, z^{-1}\right)$ that lies at the basis of the Lax equations of the AKNS hierarchy. Namely, consider the subspaces

$$
L_{\text {angl }}(\mathbb{C})_{\geqslant 0}:=\left\{\gamma \mid \gamma \in L_{\text {angl }}(\mathbb{C}), \gamma=\sum_{k=0}^{\infty} \gamma_{k} z^{k}\right\},
$$




$$
\begin{aligned}
& L_{\text {angl }}(\mathbb{C})_{<0} \\
& \quad:=\left\{\gamma \mid \gamma \in L_{\text {angl }}(\mathbb{C}), \gamma=\sum_{k=-\infty}^{-1} \gamma_{k} z^{k}\right\} .
\end{aligned}
$$

Both are Lie subalgebras of $L_{\text {angl }} \mathrm{gl}_{2}(\mathbb{C})$ and their direct sum equals the whole Lie algebra. The first Lie algebra consists of the elements in $L_{\text {an }} \mathrm{gl}_{2}(\mathbb{C})$ that extend to holomorphic maps defined on some disk $\{z \in \mathbb{C}|| z \mid \leqslant 1 / r\}, 0<r<1$, and the second Lie algebra corresponds to the maps in $L_{\text {angl }}(\mathbb{C})$ that have a holomorphic extension towards some disk around infinity $\left\{z \in \mathbb{P}^{1}(\mathbb{C})|| z \mid \geqslant r\right\}, 0<r<1$, and that are zero at infinity. To each of the two Lie subalgebras belongs a subgroup of $L_{\text {an }} \mathrm{GL}_{2}(\mathbb{C})$. The pointwise exponential map applied to elements of $L_{\text {angl }} \mathrm{gl}_{2}(\mathbb{C})_{<0}$ yields elements of

$$
U_{-}=\left\{\gamma \mid \gamma \in L_{\mathrm{angl}}(\mathbb{C}), \gamma=\mathrm{Id}+\sum_{k=-\infty}^{-1} \gamma_{k} z^{k}\right\}
$$

and the exponential map applied to elements of $L_{\text {an }} \mathrm{gl}_{2}(\mathbb{C})_{\geqslant 0}$ maps them into

$$
\begin{aligned}
P_{+} & =\left\{\gamma \mid \gamma \in L_{\text {angl }}(\mathbb{C}), \gamma=\gamma_{0}+\sum_{k=1}^{\infty} \gamma_{k} z^{k}, \text { with } \gamma_{0}\right. \\
& \left.\in \mathrm{GL}_{2}(\mathbb{C})\right\} .
\end{aligned}
$$

Both $U_{-}$and $P_{+}$are easily seen to be subgroups of $L_{\text {an }} \mathrm{GL}_{2}(\mathbb{C})$ and since the direct sum of their Lie algebras is $L_{\text {angl }}(\mathbb{C})$, their product

$$
\Omega=U_{-} P_{+}
$$

is open in $L_{\text {an }} \mathrm{GL}_{2}(\mathbb{C})$ and is called, like in the finite dimensional case, the big cell with respect to $U_{-}$and $P_{+}$.

The second decomposition is the analogue of the splitting (11) of $\mathrm{gl}_{2}(R)\left[z, z^{-1}\right)$ that led to the Lax equations of the strict AKNS hierarchy. Now we consider the subspaces

$$
\begin{aligned}
& L_{\text {angl }}(\mathbb{C})_{>0}:=\left\{\gamma \mid \gamma \in L_{\text {angl }}(\mathbb{C}), \gamma=\sum_{k=1}^{\infty} \gamma_{k} z^{k}\right\}, \\
& L_{\text {angl }}(\mathbb{C})_{\leqslant 0} \\
& :=\left\{\gamma \mid \gamma \in L_{\text {angl }}(\mathbb{C}), \gamma=\sum_{k=-\infty}^{0} \gamma_{k} z^{k}\right\} .
\end{aligned}
$$

Both are Lie subalgebras of $L_{\text {an }} \mathrm{gl}_{2}(\mathbb{C})$ and their direct sum equals the whole Lie algebra. $L_{\text {an }} \mathrm{gl}_{2}(\mathbb{C})_{>0}$ consists of maps whose holomorphic extension to $z=0$ equals zero in that point and $L_{\mathrm{an}} \mathrm{gl}_{2}(\mathbb{C})_{\leqslant 0}$ is the set of maps that extend homomorphically to a neighborhood of infinity. To each of them belongs a subgroup of $L_{\text {an }} \mathrm{GL}_{2}(\mathbb{C})$. The pointwise exponential map applied to elements of $L_{\text {angl }}(\mathbb{C})_{>0}$ yields elements of

$$
U_{+}=\left\{\gamma \mid \gamma \in L_{\text {angl }}(\mathbb{C}), \gamma=\mathrm{Id}+\sum_{k=1}^{\infty} \gamma_{k} z^{k}\right\}
$$

and the exponential map applied to elements of $L_{\mathrm{angl}} \mathrm{gl}_{2}(\mathbb{C})_{\leqslant 0}$ maps them into

$$
\begin{aligned}
P_{-} & =\left\{\gamma \mid \gamma \in L_{\mathrm{an}} \mathrm{gl}_{2}(\mathbb{C}), \gamma=\gamma_{0}\right. \\
& \left.+\sum_{k=-\infty}^{-1} \gamma_{k} z^{k}, \text { with } \gamma_{0} \in \mathrm{GL}_{2}(\mathbb{C})\right\} .
\end{aligned}
$$

Both $P_{-}$and $U_{+}$are easily seen to be subgroups of $L_{\text {an }} \mathrm{GL}_{2}(\mathbb{C})$. Since the direct sum of their Lie algebras is $L_{\text {an }} g l_{2}(\mathbb{C})$, their product $P_{-} U_{+}$is open and because $P_{+}=\mathrm{GL}_{2}(\mathbb{C}) U_{+}=$ $U_{+} \mathrm{GL}_{2}(\mathbb{C})$ and $P_{-}=\mathrm{GL}_{2}(\mathbb{C}) U_{-}=U_{-} \mathrm{GL}_{2}(\mathbb{C})$, this gives the equality

$$
P_{-} U_{+}=\Omega=U_{-} P_{+}
$$

the big cell in $L_{\text {an }} \mathrm{GL}_{2}(\mathbb{C})$.

The next two subgroups of $L_{\text {an }} \mathrm{GL}_{2}(\mathbb{C})$ correspond to the exponential factors in both linearizations. The group of commuting flows relevant for the AKNS hierarchy is the group

$$
\Gamma_{0}=\left\{\gamma_{0}(t)=\exp \left(\sum_{i=0}^{\infty} t_{i} Q_{0} z^{i}\right) \mid \gamma_{0} \in P_{+}\right\}
$$

and, similarly, for the strict AKNS hierarchy, we use the commuting flows from

$$
\Gamma_{1}=\left\{\gamma_{1}(t)=\exp \left(\sum_{i=1}^{\infty} t_{i} Q_{0} z^{i}\right) \mid \gamma_{1} \in U_{+}\right\} .
$$

Besides the groups $\Delta, \Gamma_{0}$, and $\Gamma_{1}$, there are more subgroups in $L_{\text {an }} \mathrm{GL}_{2}(\mathbb{C})$ that commute with those three groups and they are in a sense complimentary to $\Delta, \Gamma_{0}$ and $\Delta, \Gamma_{1}$, respectively. That is why we use the following notations for them: in $U_{-}$ there is

$$
\Gamma_{0}^{c}=\left\{\gamma_{0}^{c}(t)=\exp \left(\sum_{k=1}^{\infty} s_{k} Q_{0} z^{-k}\right) \mid \gamma_{0}^{c} \in U_{-}\right\}
$$

and in $P_{-}$we have

$$
\Gamma_{1}^{c}=\left\{\gamma_{1}^{c}(t)=\exp \left(\sum_{k=0}^{\infty} s_{k} Q_{0} z^{-k}\right) \mid \gamma_{1}^{c} \in P_{-}\right\} .
$$

We have now all ingredients to describe the construction of the solutions to each hierarchy and we start with the AKNS hierarchy. Take inside the product $L_{\text {an }} \mathrm{GL}_{2}(\mathbb{C}) \times \Delta$ the collection $S_{0}$ of pairs $(g, \delta(m))$ such that there exists a $\gamma_{0}(t) \in \Gamma_{0}$ satisfying

$$
\delta(m) \gamma_{0}(t) g \gamma_{0}(t)^{-1} \delta(-m) \in \Omega=U_{-} P_{+} .
$$

For such a pair $(g, \delta(m))$, we take the collection $\Gamma_{0}(g, \delta(m))$ of all $\gamma_{0}(t)$ satisfying the condition (77). This is an open nonempty subset of $\Gamma_{0}$. Let $R_{0}(g, \delta(m))$ be the algebra of analytic functions $\Gamma_{0}(g, \delta(m)) \rightarrow \mathbb{C}$. This is the algebra of 
functions $R$ that we associate with the point $(g, \delta(m)) \in S_{0}$ and for the commuting derivations of $R_{0}(g, \delta(m))$ we choose $\partial_{i}:=\partial / \partial t_{i}, i \geqslant 0$. By property (77), we have for all $\gamma_{0}(t) \epsilon$ $\Gamma_{0}(g, \delta(m))$

$$
\begin{aligned}
& \delta(m) \gamma_{0}(t) g \gamma_{0}(t)^{-1} \delta(-m) \\
& =u_{-}(g, \delta(m))^{-1} p_{+}(g, \delta(m)),
\end{aligned}
$$

with $u_{-}(g, \delta(m)) \in U_{-}, p_{+}(g, \delta(m)) \in P_{+}$. Then, all the matrix coefficients in the Fourier expansions of the elements $u_{-}(g$, $\delta(m))$ and $p_{+}(g, \delta(m))$ belong to the algebra $R_{0}(g, \delta(m))$. It is convenient to write relation (78) in the form

$$
\begin{aligned}
\Psi_{g, \delta(m)} & :=u_{-}(g, \delta(m)) \delta(m) \gamma_{0}(t) \\
& =p_{+}(g, \delta(m)) \delta(m) \gamma_{0}(t) g^{-1} \\
& =p_{+}(g, \delta(m)) \gamma_{0}(t) \delta(m) g^{-1} \\
& =q_{+}(g, \delta(m)) \delta(m) g^{-1},
\end{aligned}
$$

with $q_{+}(g, \delta(m)) \in P_{+}$. Clearly, $\Psi_{g, \delta(m)}$ is an oscillating matrix of type $\delta(m)$ in $\mathscr{M}_{0}$, where all the products in the decomposition $u_{-}(g, \delta(m)) \delta(m) \gamma_{0}(t)$ are no longer formal but real. This is our potential wave matrix for the AKNS hierarchy.

To get the potential wave function for the strict AKNS hierarchy, we proceed similarly; only this time, we use the decomposition $\Omega=P_{-} U_{+}$. Consider again the product $L_{\text {an }} \mathrm{GL}_{2}(\mathbb{C}) \times \Delta$ and the subset $S_{1}$ of pairs $(g, \delta(m))$ such that there exists a $\gamma_{1}(t) \in \Gamma_{1}$ satisfying

$$
\delta(m) \gamma_{1}(t) g \gamma_{1}(t)^{-1} \delta(-m) \in \Omega=P_{-} U_{+} .
$$

For such a pair $(g, \delta(m))$, we define $\Gamma_{1}(g, \delta(m))$ as the set of all $\gamma_{1}(t)$ satisfying condition (77). This is an open nonempty subset of $\Gamma_{1}$. Let $R_{1}(g, \delta(m))$ be the algebra of analytic functions $\Gamma_{1}(g, \delta(m)) \rightarrow \mathbb{C}$. This is the algebra of functions $R$ that we associate with the point $(g, \delta(m)) \in S_{1}$ and for the commuting derivations of $R_{1}(g, \delta(m))$ we choose $\partial_{i}:=\partial / \partial t_{i}$, $i>0$. By property (80), we have for all $\gamma_{1}(t) \in \Gamma_{1}(g, \delta(m))$

$$
\begin{aligned}
& \delta(m) \gamma_{1}(t) g \gamma_{1}(t)^{-1} \delta(-m) \\
& =p_{-}(g, \delta(m))^{-1} u_{+}(g, \delta(m)),
\end{aligned}
$$

with $p_{-}(g, \delta(m)) \in P_{-}, u_{+}(g, \delta(m)) \in U_{+}$. Then, all the matrix coefficients in the Fourier expansions of the elements $p_{-}(g, \delta(m))$ and $u_{+}(g, \delta(m))$ belong to the algebra $R_{1}(g, \delta(m))$. It is convenient to write relation (81) in the form

$$
\begin{aligned}
\Phi_{g, \delta(m)}:=p_{-}(g, \delta(m)) \delta(m) \gamma_{1}(t) \\
=u_{+}(g, \delta(m)) \delta(m) \gamma_{1}(t) g^{-1} \\
=u_{+}(g, \delta(m)) \gamma_{1}(t) \delta(m) g^{-1} \\
=w_{+}(g, \delta(m)) \delta(m) g^{-1},
\end{aligned}
$$

with $w_{+}(g, \delta(m)) \in U_{+}$. Clearly, $\Phi_{g, \delta(m)}$ is an oscillating matrix of type $\delta(m)$ in $\mathscr{M}_{1}$ where all the products in the decomposition $p_{-}(g, \delta(m)) \delta(m) \gamma_{1}(t)$ are no longer formal but real.

To show that $\Psi_{g, \delta(m)}$ is a wave matrix for the AKNS hierarchy of type $\delta(m)$ and $\Phi_{g, \delta(m)}$ one for the strict AKNS hierarchy, we use the fact that it suffices to prove the relations in Proposition 6. We treat first $\Psi_{g, \delta(m)}$. In (79), we presented two different decompositions of $\Psi_{g, \delta(m)}$ that we will use to compute $\partial_{i}\left(\Psi_{g, \delta(m)}\right)$. With respect to $u_{-}(g, \delta(m)) \delta(m) \gamma_{0}(t)$, there holds

$$
\begin{aligned}
\partial_{i}( & \left.\Psi_{g, \delta(m)}\right) \\
= & \left\{\partial_{i}\left(u_{-}(g, \delta(m))\right) u_{-}(g, \delta(m))^{-1}+z^{i}\right\} \\
& \cdot u_{-}(g, \delta(m)) \delta(m) \gamma_{0}(t)=M_{i} \Psi_{g, \delta(m)},
\end{aligned}
$$

with $M_{i} \in \operatorname{gl}_{2}(R(g, \delta(m)))\left[z, z^{-1}\right)$ of order $i$ in $z$. If we take the decomposition $q_{+}(g, \delta(m)) \delta(m) g^{-1}$, then we get

$$
\begin{aligned}
\partial_{i} & \left(\Psi_{g, \delta(m)}\right) \\
& =\left\{\partial_{i}\left(q_{+}(g, \delta(m))\right) q_{+}(g, \delta(m))^{-1}\right\} \Psi_{g, \delta(m)}
\end{aligned}
$$

with $\partial_{i}\left(q_{+}(g, \delta(m))\right) q_{+}(g, \delta(m))^{-1}$ of the form $\sum_{j \geqslant 0} q_{j} z^{j}$, with all $q_{j} \in \mathrm{gl}_{2}(R(g, \delta(m)))$. As $\Psi_{g, \delta(m)}$ is invertible, we obtain $M_{i}=\sum_{j \geqslant 0} q_{j} z^{j}$ and thus $M_{i}=\sum_{j=0}^{i} q_{j} z^{j}$ and that is the desired result.

We handle the case of $\Phi_{g, \delta(m)}$ in a similar way. Also in (79), you can find two different decompositions of $\Phi_{g, \delta(m)}$ that we will use to compute $\partial_{i}\left(\Phi_{g, \delta(m)}\right)$. With respect to the expression $p_{-}(g, \delta(m)) \delta(m) \gamma_{1}(t)$ we get for all $i \geqslant 1$

$$
\begin{aligned}
\partial_{i}\left(\Phi_{g, \delta(m)}\right) & \\
= & \left\{\partial_{i}\left(p_{-}(g, \delta(m))\right) p_{-}(g, \delta(m))^{-1}+z^{i}\right\} \\
& \cdot p_{-}(g, \delta(m)) \delta(m) \gamma_{1}(t)=N_{i} \Phi_{g, \delta(m)},
\end{aligned}
$$

again with $N_{i} \in \operatorname{gl}_{2}\left(R_{1}(g, \delta(m))\right)\left[z, z^{-1}\right)$ of order $i$ in $z$. Next we take the decomposition $w_{+}(g, \delta(m)) \delta(m) g^{-1}$ and that yields

$$
\begin{aligned}
\partial_{i} & \left(\Phi_{g, \delta(m)}\right) \\
& =\left\{\partial_{i}\left(w_{+}(g, \delta(m))\right) w_{+}(g, \delta(m))^{-1}\right\} \Psi_{g, \delta(m)} .
\end{aligned}
$$

Since the zeroth order term of $w_{+}(g, \delta(m))$ is Id, we note that the element $\partial_{i}\left(w_{+}(g, \delta(m))\right) w_{+}(g, \delta(m))^{-1}$ has the form $\sum_{j \geqslant 1} w_{j} z^{j}$, with all $w_{j} \in \mathrm{gl}_{2}\left(R_{1}(g, \delta(m))\right)$. As $\Phi_{g, \delta(m)}$ is also invertible, we obtain $N_{i}=\sum_{j \geqslant 1} w_{j} z^{j}$ and thus $N_{i}=\sum_{j=1}^{i} w_{j} z^{j}$ and that is the result we were looking for in the strict case.

The abovementioned constructions are not affected seriously by a number of transformations. For example, if we write $\delta=\delta((1,1))$, then all $\delta^{k}, k \in \mathbb{Z}$, are central elements and we have for all $(g, \delta(m)) \in S_{0}$ and $S_{1}$ respectively and all $k \in \mathbb{Z}$

$$
\begin{aligned}
\Psi_{g, \delta(m) \delta^{k}} & =\Psi_{g, \delta(m)} \delta^{k} \\
\text { respectively } \Phi_{g, \delta(m) \delta^{k}} & =\Phi_{g, \delta(m)} \delta^{k} .
\end{aligned}
$$


Also for any $p_{1} \in P_{+}$, each $\gamma_{0}^{c}(s) \in \Gamma_{0}^{c}$, and all $(g, \delta(m)) \in$ $S_{0}$, we see that the element $\left(\gamma_{0}^{c}(s) g \delta(-m) p_{1} \delta(m), \delta(m)\right)$ also belongs to $S_{0}$, for

$$
\begin{aligned}
& \delta(m) \gamma_{0}(t) \gamma_{0}^{c}(s) g \delta(-m) p_{1} \delta(m) \gamma_{0}(t)^{-1} \delta(-m) \\
& =\gamma_{0}^{c}(s) u_{-}^{-1} p_{+} \gamma_{0}(t) p_{1} \gamma_{0}(t)^{-1} .
\end{aligned}
$$

In particular, we see that

$$
\Psi_{\gamma_{0}^{c}(s) g \delta(-m) p_{1} \delta(m), \delta(m)}=u_{-} \gamma_{0}^{c}(s)^{-1} \delta(m) \gamma_{0}(t) .
$$

Its analogue in the strict case is as follows: for any $u_{1} \in$ $U_{+}$, each $\gamma_{1}^{c}(s) \in \Gamma_{1}^{c}$, and all $(g, \delta(m)) \in S_{1}$, the element $\left(\gamma_{1}^{c}(s) g \delta(-m) u_{1} \delta(m), \delta(m)\right)$ also belongs to $S_{1}$ and there holds

$$
\Phi_{\gamma_{1}^{c}(s) g \delta(-m) u_{1} \delta(m), \delta(m)}=p_{-} \gamma_{1}^{c}(s)^{-1} \delta(m) \gamma_{1}(t)
$$

We resume the foregoing results in the following theorem.

Theorem 7. Consider the product space $\Pi:=L_{\text {an }} \mathrm{GL}_{2}(\mathbb{C}) \times \Delta$. Then, the following holds:

(a) For each point $(g, \delta(m)) \in \Pi$ that satisfies condition (77), we have a wave matrix of type $\delta(m)$ of the AKNS hierarchy $\Psi_{g, \delta(m)}$, defined by (79), and this determines a solution $Q_{g, \delta(m)}=u_{-}(g, \delta(m)) Q_{0} u_{-}(g, \delta(m))^{-1}$ of the AKNS hierarchy. For each $p_{1} \in P_{+}$, every $\gamma_{0}^{c}(s) \in \Gamma_{0}^{c}$, and all powers of $\delta$, one has

$$
Q_{\gamma_{0}^{c}(s) g \delta(-m) p_{1} \delta(m), \delta^{k} \delta(m)}=Q_{g, \delta(m)} .
$$

(b) For each point $(g, \delta(m)) \in \Pi$ that satisfies condition (80), we have a wave matrix of type $\delta(m)$ of the strict AKNS hierarchy $\Phi_{g, \delta(m)}$, defined by (82), and it determines a solution $Z_{g, \delta(m)}=p_{-}(g, \delta(m)) Q_{0} z p_{-}(g$, $\delta(m))^{-1}$ of the strict AKNS hierarchy. For each $u_{1} \in U_{+}$, every $\gamma_{1}^{c}(s) \in \Gamma_{1}^{c}$, and all powers of $\delta$, one has

$$
Z_{\gamma_{1}^{c}(s) g \delta(-m) u_{1} \delta(m), \delta^{k} \delta(m)}=Z_{g, \delta(m)} .
$$

\section{Competing Interests}

The author declares that they have no competing interests.

\section{References}

[1] M. Adler, P. van Moerbeke, and P. Vanhaecke, Algebraic Integrability, Painlevé Geometry and Lie Algebras, vol. 47 of Ergebnisse der Mathematik und ihrer Grenzgebiete, Springer, 2004.

[2] G. F. Helminck, A. G. Helminck, and E. A. Panasenko, "Integrable deformations in the algebra of pseudodifferential operators from a Lie algebraic perspective," Theoretical and Mathematical Physics, vol. 174, no. 1, pp. 134-153, 2013.

[3] M. J. Ablowitz, D. J. Kaup, A. C. Newell, and H. Segur, "The inverse scattering transform-Fourier analysis for nonlinear problems," Studies in Applied Mathematics, vol. 53, no. 4, pp. 249-315, 1974.
[4] H. Flashka, A. C. Newell, and T. Ratiu, "Kac-Moody Lie algebras and soliton equations II. Lax equations associated with $A_{1}{ }^{(1)}$," Physica D: Nonlinear Phenomena, vol. 9, no. 3, pp.300-323, 1983.

[5] E. Date, M. Jimbo, M. Kashiwara, and T. Miwa, "Transformation groups for soliton equations," in Proceedings of the RIMS Symposium on Nonlinear Integrable Systems-Classical Theory and Quantum Theory, T. Jimbo and T. Miwa, Eds., World Science Publisher, Kyoto, Japan, 1981.

[6] G. Segal and G. Wilson, "Loop groups and equations of KdV type," Publications Mathématiques de l'IHÉS, vol. 61, pp. 5-65, 1985.

[7] A. Pressley and G. Segal, Loop Groups, Oxford Mathematical Monographs, Clarendon Press, 1986.

[8] R. S. Hamilton, "The inverse function theorem of Nash and Moser," Bulletin of the American Mathematical Society, vol. 7, no. 1, pp. 65-222, 1982. 


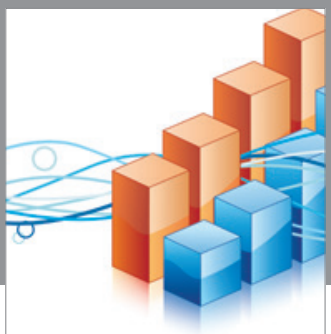

Advances in

Operations Research

vatem alat4

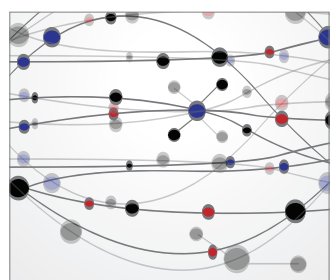

\section{The Scientific} World Journal
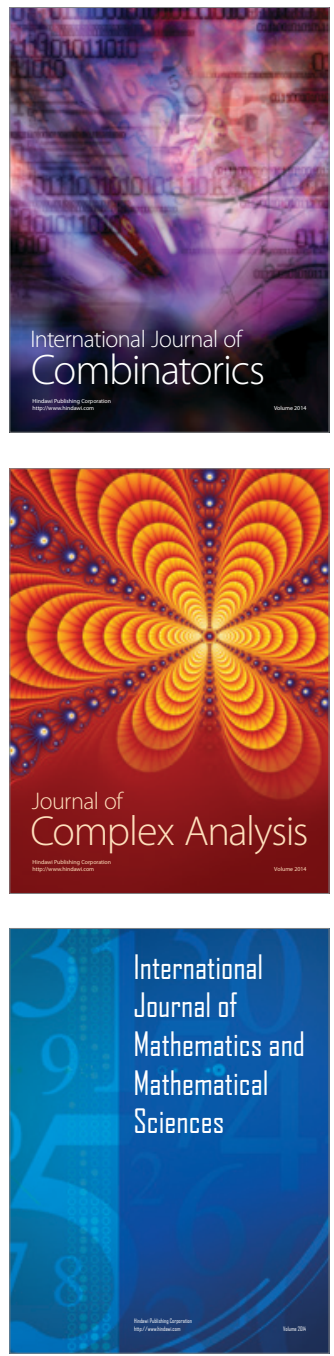
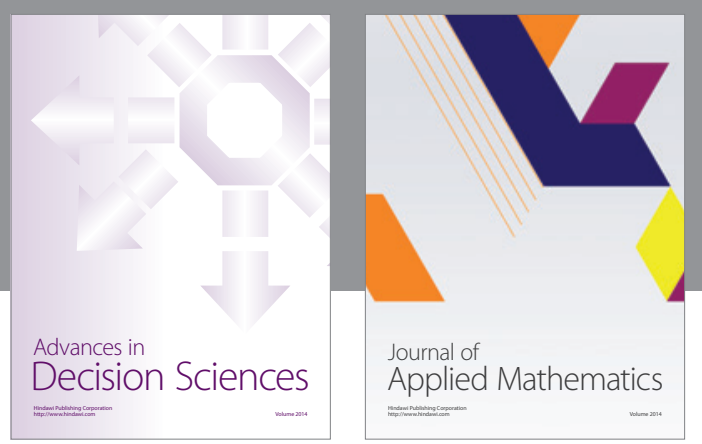

Algebra

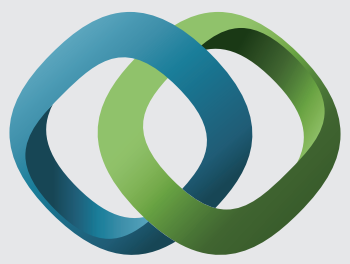

\section{Hindawi}

Submit your manuscripts at

http://www.hindawi.com
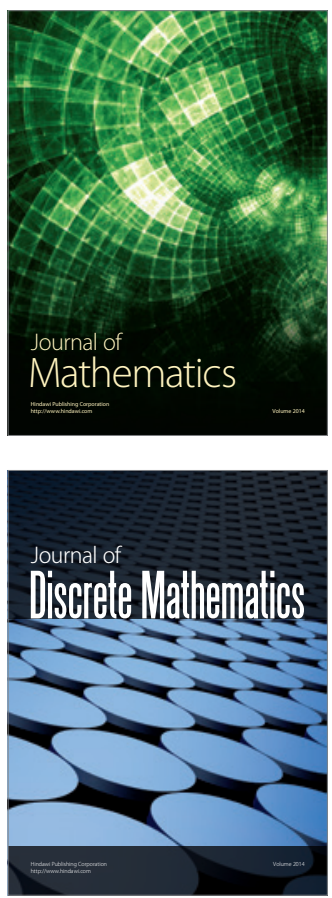

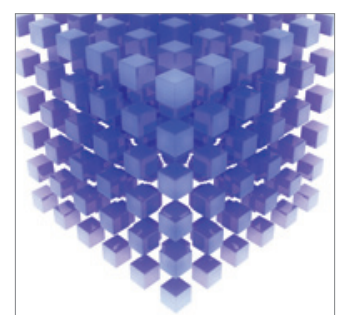

Mathematical Problems in Engineering
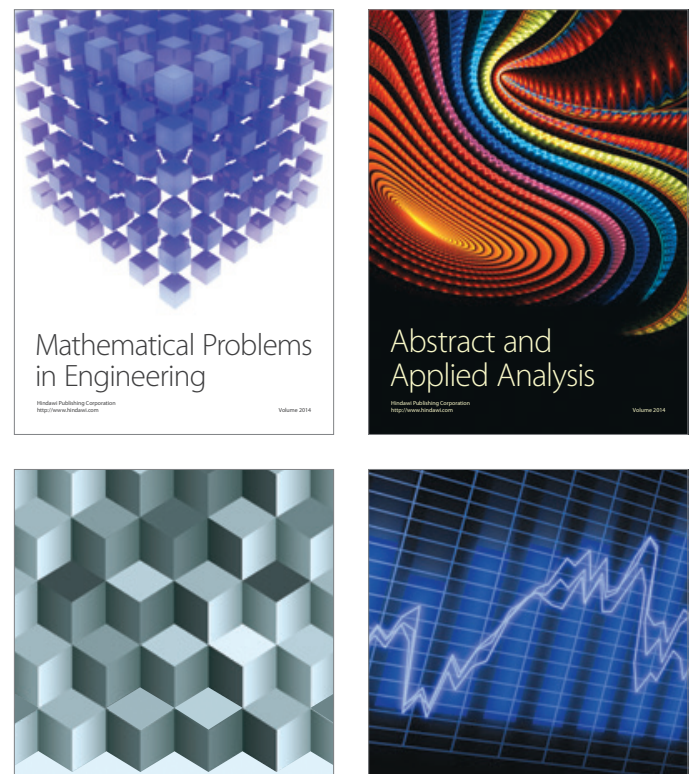

Journal of

Function Spaces

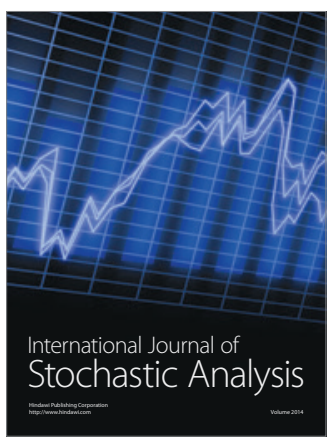

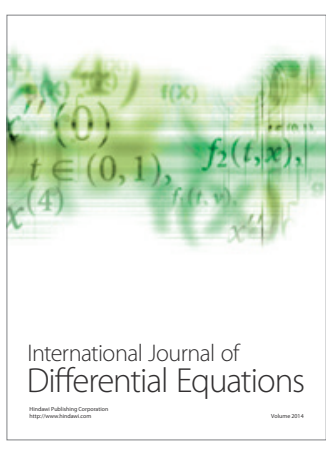
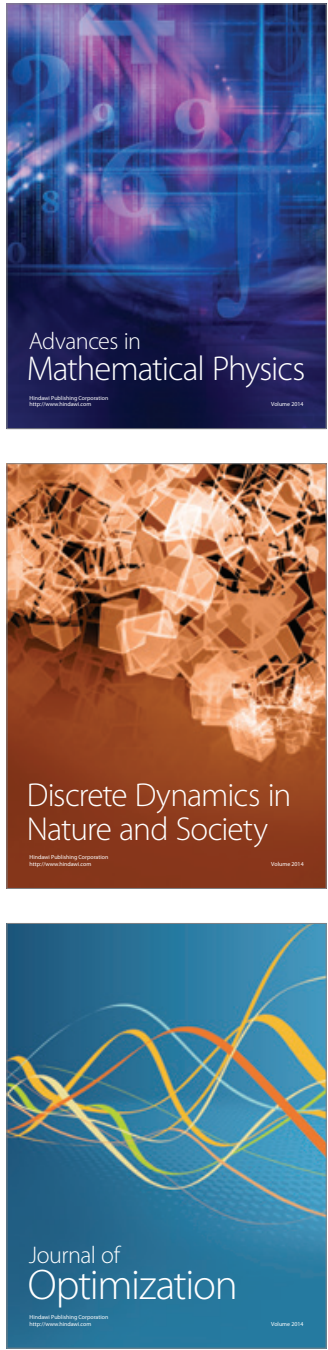